\title{
A REPRESENTATION-THEORETIC CRITERION FOR LOCAL SOLVABILITY OF LEFT INVARIANT DIFFERENTIAL OPERATORS ON NILPOTENT LIE GROUPS \\ BY
}

\author{
LAWRENCE CORWIN ${ }^{1}$
}

\begin{abstract}
Let $L$ be a left invariant differential operator on the nilpotent Lie group $N$. It is shown that if $\pi(L)$ is invertible for all irreducible representations $\pi$ in general position (and if the inverses satisfy some mild technical conditions), then $L$ is locally solvable. This result genera'izes a theorem of $\mathrm{L}$. Rothschild.
\end{abstract}

1. Let $N$ be a connected, simply connected nilpotent Lie group, with Lie algebra $\Re$, and let $L$ be an element of the enveloping algebra $U(\Re)$ of $\Re$. We may regard $L$ as a left invariant operator on $N$ acting by convolution; that is, we consider $L$ as a distribution whose support is the identity, and let $\phi \rightarrow \phi * L$ be the operator associated with $L$. Similarly, $\phi \rightarrow L * \phi$ is the right invariant operator associated with $L$. (This is not the standard convention; see $\$ 4$ for a discussion.) If $\pi$ is any unitary representation of $N$, then we may define $\pi(L)$; for $L \in \Re, \pi(L) v=$ $\left.(d / d t)(\pi(\exp t L) v)\right|_{t=0}$. In general, $\pi(L)$ is an unbounded operator. One advantage of the definitions given above is that, on appropriate domains,

$$
\pi(\phi * L)=\pi(\phi) \pi(L), \quad \pi(L * \phi)=\pi(L) \pi(\phi) .
$$

In [10], Rothschild gave a representation-theoretic condition that implied local solvability for certain left invariant operators $L$ on the $(2 n+1)$-dimensional Heisenberg group, $H_{n}$. $L$ was required to be homogeneous with respect to certain dilations on the group; her main theorem said that $L$ was locally solvable if $\pi(L)$ had a bounded right inverse (an operator $B_{\pi}$ with $\pi(L) B_{\pi}=I$ ) for all infinite-dimensional representations $\pi$ of $H_{n}$. (The theorem refers to left inverses, but the proof makes it clear that right inverses are required.) Rockland had conjectured such a result in [9].

In this note, we generalize this theorem to arbitrary (connected, simply connected) nilpotent Lie groups. In this generality, we need to make two further hypotheses, one about the smoothness of the inverse as the representations vary and one giving bounds on the norms of the inverses. Both hypotheses are trivially satisfied by the operators in [10].

In the next section, we give some results about Plancherel measure and about central measures in $U(\mathscr{N})$; they are used in the proof of the main theorem, given in §3. $\$ 4$ is devoted to assorted remarks.

Received by the editors January 24, 1980 and, in revised form, March 11, 1980.

1980 Mathematics Subject Classification. Primary 22E30, 58G15; Secondary 22E25, 22E45, 35 A05.

'Supported by NSF Contract NSF MCS 78-02715. 
2. The results given here are not new; most are proved in [4], [6], or [7], though perhaps not quite in the same form.

$N$ acts on $\Re$ by the adjoint map, and on $\Re^{*}$ by the coadjoint map, Ad*. The irreducible unitary representations of $N$ are known, from [4], to be in 1-1 correspondence with the $\mathrm{Ad}^{*}$-orbits on $\varkappa^{*}$. Parametrizing all these orbits can be a complicated matter, but there is a relatively simple parametrization of "almost all" the orbits, and this will suffice for our purposes.

Let $X_{1}, \ldots, X_{n}$ be a "strong Malcev basis" for $\Re$ (i.e., a basis such that $\Re_{j}=\operatorname{span}\left\{X_{1}, \ldots, X_{j}\right\}$ is an ideal of $\mathscr{N}, \forall j \leqslant n$ ). We may assume that Lebesgue measure has been so normalized that $\left\{\sum_{j=1}^{n} a_{j} X_{j}: 0<a_{j}<1,1<j<n\right\}$ has volume 1. (When we deal with subspaces spanned by subsets of the $X_{j}$, we normalize Lebesgue measure similarly.) Let $l_{1}, \ldots, l_{n}$ be the dual basis on $\mathfrak{l}^{*}$.

THeOrem 1. There are complementary subsets $S, T$ of $\{1, \ldots, n\}$, plus a Zariskiopen dense subset $\mathcal{V}$ of $V_{2}=\operatorname{span}\left\{l_{j}: j \in T\right\}$, such that if $V_{1}=\operatorname{span}\left\{l_{j}: j \in S\right\}$, then

(1) for almost every $\operatorname{Ad}^{*}(N)$-orbit $\theta \subset \Re^{*}, \theta \cap V_{2}$ has only one element (if this is $l$, we let $\theta=\theta_{l}$ );

(2) $\left\{l: \exists \vartheta_{1}\right.$ such that $\left.\{l\}=\vartheta_{l} \cap V_{2}\right\} \supseteq \mathcal{V}$;

(3) for all $l \in \mathcal{V}$, there is a polynomial $P_{l}: V_{1} \rightarrow V_{2}$ with $\theta_{l}=$ graph $P_{l}$;

(4) the map $l \rightarrow P_{l}$ is rational in $l$.

("Almost every orbit" means "enough orbits for the Plancherel measure;" in fact, the set of orbits $\mathcal{O}_{l}, l \in \mathcal{V}$, fills a nonempty Zariski-open subset of $\mathfrak{\Re}$.)

Proof. This result is essentially that on p. 55 of [6].

From the theorem, $\mathcal{V}$ parametrizes a subset of $N$ which carries Plancherel measure. A representation in this subset will be said to be in general position; we shall consider only such orbits unless we specify otherwise. (Note: the definition of general position can depend on the basis $X_{1}, \ldots, X_{n}$.) We generally regard Plancherel measure as defined on $\mathfrak{V}$ (or $V_{2}$ ). We specify Lebesgue measure on $V$ to be normalized such that $\left\{\sum_{j=1}^{n} a_{j} l_{j}: 0<a_{j}<1,1<j<n\right\}$ has volume 1 , and normalize Lebesgue measure on $V_{1}, V_{2}$ similarly.

The element $l$ defines an antisymmetric bilinear form $B_{l}$ on $\Re$ by

$$
B_{l}(X, Y)=l([X, Y]) \text {. }
$$

The radical of this form, $\mathcal{R}_{l}$, is a subalgebra of $\Re$, and

$$
\Re_{l}=T_{l}\left(\Theta_{l}\right)^{\perp}
$$

the annihilator of the tangent space to $\theta_{l}$ at $l$. (We have regarded $\theta_{l}$ as a submanifold of $\mathscr{X}^{*}$ and identify the tangent and cotangent spaces to $\Re^{*}$ at $l$ with $\Re^{*}, \Re$ respectively.) Let $\Re_{1}=\operatorname{span}\left\{X_{j}: j \in S\right\}, \Re_{2}=\operatorname{span}\left\{X_{j}: j \in T\right\}$; let $P$ be the projection of $\mathscr{N}$ onto $\Re_{2}$ which annihilates $\Re_{1}$.

LemMa 1. For $l \in \mathcal{V},\left.P\right|_{\Re_{l}}$ is a 1-1 map of $\Re_{l}$ onto $\Re_{2}$, and $\Re_{1}$ is a cross-section for $\Re_{l} \backslash \Re$. 
Proof. Since $\operatorname{dim} \Re_{l}=\operatorname{codim} T_{l}\left(\Theta_{l}\right)=\operatorname{codim} V_{1}=\operatorname{dim} \mathscr{T}_{2}$, the first claim follows once we show that ker $\left.P\right|_{\Re_{1}}$ is trivial. If $X \in \Re_{l} \cap \operatorname{ker} P=\Re_{l} \cap \Re_{2}$, then $X$ annihilates $V_{2}$ and $T_{l}\left(\theta_{l}\right)$. Property (3) of Theorem 1 shows that $V$, and $T_{l}\left(\theta_{l}\right)$ are transverse, and dimension counting shows that $\Re^{*}=V_{2}+T_{l}\left(\theta_{l}\right)$. Thus $X=0$. The second half is essentially the dual of the first.

LEMma 2. The $\operatorname{Ad}^{*}(N)$-invariant measures on $\theta_{l}$ are multiples of the measure obtained by lifting Lebesgue measure on $V_{1}$ to $\theta_{l}=$ graph $P$.

Proof. This is proved on p. 54 of [6].

Let $\operatorname{Pf}(l)=$ Pfaffian of $\left.B_{l}\right|_{\Re_{1}} \cdot \operatorname{Pf}(l)$ is a polynomial function of $l$; if $l \in \mathcal{T}$, then, since $B_{l}$ is nonsingular in $\Re_{l} \mid \mathscr{T}_{1}, \operatorname{Pf}(l) \neq 0$ (see Lemma 1 ).

Now let $\phi \in \mathcal{S}(N)$, the space of Schwartz class functions on $N$. Then $\phi \circ \exp \in$ $S(\Re)$, and we may define $\phi^{\wedge}$ on $\mathcal{R}^{*}$ by

$$
\phi^{\prime}\left(l^{\prime}\right)=\int_{\Re}(\phi \circ \exp )(X) \exp \left(2 \pi i l^{\prime}(x)\right) d x .
$$

According to Theorem 7.3 of [4], the operator $\pi_{l}(\phi)$ (where $\pi_{l}$ is the irreducible representation corresponding to $\left.\theta_{l}\right)$ is a trace class operator, and there is a "canonical measure" $\mu_{l}$ defined on $\mathcal{\theta}_{l}$ such that for all $l \in \mathcal{V}$ and all $\phi \in \mathcal{S}(N)$,

$$
\operatorname{Tr} \pi_{l}(\phi)=\int_{\theta_{l}} \phi^{\wedge}\left(l^{\prime}\right) d \mu_{l}\left(l^{\prime}\right)
$$

The measure $\mu_{l}$ is $\operatorname{Ad}(N)$-invariant. If we let $\nu_{l}$ be the pullback to $\theta_{l}$ of the normalized Lebesgue measure $d_{1} l$ on $V_{1}$, then $\mu_{l}$ is a multiple of $\nu_{l}$ (see Lemma 2).

THEOREM 2. $\mu_{l}=|\operatorname{Pf}(l)|^{-1} \nu_{l}$.

Proof. The Lebesgue measures on $\Re_{1}$ and $V_{1}$ have been chosen to be dual (for the Fourier transform).

Suppose that the elements of $S$ are (in ascending order) $j_{1}, \ldots, j_{n}$. Let

$$
F\left(a_{1} X_{1}+\cdots+a_{k} X_{k}\right)=\exp a_{k} X_{j_{k}} \cdots \exp a_{1} X_{j_{1}} \quad\left(a_{1}, \ldots, a_{k} \in \mathbf{R}\right),
$$

so that $F: \Re_{1} \rightarrow N$; let $N_{1}=F\left(\Re_{1}\right) . N_{1}$ is not necessarily a group, but it is a cross-section for $N / \mathscr{R}(l)$, and therefore the map $G: \Re_{1} \rightarrow \Re^{*}$, defined by $G(x)=\operatorname{Ad}^{*} F(x)(l)$, maps $G$ diffeomorphically onto $\vartheta_{l}$; moreover, it takes $d_{1} X$, the Haar measure on $\Re_{1}$, to a multiple of $\mu_{l}$. We write $\mu_{l}=C_{l} G_{*}\left(d_{1} X\right)$. In fact, $C_{l}$ is defined by the property that $C_{l} d_{1} X$ is self-dual when we identify $\Re_{1}$ with its dual via $B_{1}$. (These facts are proved in Lemma 5.1 of [1].)

If $P^{\prime}$ is the projection of $\Re^{*}$ to $V_{1}$ with kernel $V_{2}$, then $P^{\prime} \circ G: \Re^{*} \rightarrow V_{1}$, and

$$
d\left(P^{\prime} \circ G\right)=\sum_{k \in S} c_{j k} l_{k}
$$

where $c_{j k}=\left(\operatorname{Ad}^{*} X_{j}\right)(l)\left(X_{k}\right)=l\left(\left[X_{k}, X_{j}\right]\right) . P^{\prime} \circ G$ takes $d_{1} X$ to a multiple of $d_{1} l$, Lebesgue measure on $V_{1}$, and the above calculation shows that it takes the unit cube in $\Re_{1}$ to a region with volume $\left|\operatorname{Det}\left(c_{j k}\right)\right|=P f(l)^{2}$. That is,

$$
\left(P^{\prime} \circ G\right)_{*}\left(d_{l} X\right)=\operatorname{Pf}(l)^{-2} d_{1} l, \quad \text { or } \quad G_{*}\left(d_{l} X\right)=\operatorname{Pf}(l)^{-2} \nu_{l}
$$


But $C_{l}=|\operatorname{Pf}(l)| ;$ see the remark on p. 270 of [7]. Thus

$$
\mu_{l}=C_{l} G_{*}\left(d_{l} X\right)=|\operatorname{Pf}(l)|^{-1} \nu_{l},
$$

as claimed.

COROllary. Let $d_{2}^{\prime} l$ be the choice of Lebesgue measure on $V_{2}$ described earlier; then the Plancherel measure on $V_{2}$ is $|\operatorname{Pf}(l)| d_{2}^{\prime} l$.

Proof. The Plancherel measure $\nu$ is defined by

$$
\phi(e)=\int_{\hat{N}} \operatorname{Tr}(\pi(\phi)) d \nu(\phi), \quad \forall \phi \in \mathcal{S}(N)
$$

But

$$
\begin{aligned}
\phi(e) & =\phi \circ \exp (0)=\int_{V_{2}} \int_{V_{1}} \phi^{\wedge}\left(l_{1}+l\right) d_{1}\left(l_{1}\right) d_{2}(l) \\
& =\int_{V_{2}} \int_{V_{1}} \phi^{\wedge}\left(P_{l}\left(l_{1}\right)+l\right) d_{1}\left(l_{1}\right) d_{2}(l) \\
& =\int_{\mathscr{V}} \int_{V_{1}} \phi^{\wedge}\left(P_{l}\left(l_{1}\right)+l\right) \frac{|\operatorname{Pf}(l)|}{|\operatorname{Pf}(l)|} d_{1}\left(l_{1}\right) d_{2}(l) \\
& =\int_{\mathscr{V}}|\operatorname{Pf}(l)| \operatorname{Tr} \pi_{l}(\phi) d_{2}(l),
\end{aligned}
$$

and the result follows.

Note. If $\phi \in \delta(N)$, then the Fourier inversion formula,

$$
\phi(x)=\int|\operatorname{Pf}(l)| \operatorname{Tr}\left(\pi_{l}\left(x^{-1}\right) \pi_{l}(\phi)\right) d_{2}(l)
$$

and the Plancherel formula,

$$
\|\phi\|_{2}^{2}=\int_{\mathscr{V}}|\operatorname{Pf}(l)|\left\|\pi_{l}(\phi)\right\|_{2}^{2} d_{2}(l)
$$

(where $\left\|\pi_{l}(\phi)\right\|_{2}$ is the Hilbert-Schmidt norm) both hold. Moreover, $\left\|\pi_{l}(\phi)\right\|$ decreases more rapidly at $\infty$ than any nonzero rational function in $l$.

We conclude this section with some remarks about the center of $\mathscr{U}(\mathscr{N})$. Theorem 4.8.12 of [2] states that $\mathscr{Z}(\mathcal{U}(\mathscr{N})) \simeq \delta(\Re)^{N}=\operatorname{Ad}(N)$-invariant elements of $\delta(\mathscr{T})$; thus we may regard elements of $\mathscr{Z}(\mathcal{U}(\mathscr{T})$ ) as Ad*-invariant polynomials on $\mathfrak{X}^{*}$. For $l \in \mathfrak{l}^{*}$, let $\pi_{l}$ be the irreducible representation corresponding to $l$. If $A \in \mathscr{Z}(\mathcal{Q}(\mathcal{\Re})), \pi_{l}(A)=A(2 \pi i l)$. (See [3], [4], or [5].)

Lemma 3. There is an element $A \in \mathscr{Z}(\mathcal{U}(\mathscr{T}))$ such that $\pi_{l}(A)=\operatorname{Pf}(l), V l \in \mathscr{V}$.

Proof. $\operatorname{Pf}(l)$ is a homogeneous polynomial (of degree $k$, say) on $\Re^{*}$, invariant under $\mathrm{Ad}^{*}$ (see Partie 2, $\$ 2.5$ of [6]). Thus $A_{0} \in \mathscr{Z}\left(\mathcal{Q}(\mathscr{T})\right.$ ): $A_{0}(l)=\operatorname{Pf}(l)$. Let $A=(2 \pi i)^{-k} A_{0}$. 
3. We are now ready for the main result of this paper.

THEOREM 3. Let $L \in \mathcal{U}(\mathcal{N})$ be such that

(a) for every $l \in \mathfrak{V}, \pi_{l}(L)$ has a bounded left inverse, $B_{l}$;

(b) one can choose the $B_{l}$ so that they vary measurably with l;

(c) there are constants $C$ and $k$ such that for all $l \in \mathcal{V}$,

$$
\left\|B_{l}\right\| \leqslant C\left(|\operatorname{Pf}(l)|^{k}+|\operatorname{Pf}(l)|^{-k}\right) .
$$

Then $L$ is locally solvable.

Proof. (This proof follows those in [9] and [10], with a few modifications.) Let $A$ be the operator given by Lemma 3 . Then $A^{k}$ is locally solvable (see [8]), and it therefore certainly suffices to show that the equation

$$
u * L=A^{k} * \phi
$$

has a solution in $C^{\infty}(N)$ whenever $\phi \in C_{c}^{\infty}(N)$. We reason heuristically as follows: if $u \in \mathcal{L}^{1}(N)$, then $\pi_{l}(u) \pi_{l}(L)=\pi_{l}\left(A^{k}\right) \pi_{l}(\phi)$, and we should thus try to find $u$ such that

$$
\pi_{l}(u)=\pi_{l}\left(A^{k}\right) \pi_{l}(\phi) B_{l}=\operatorname{Pf}(l)^{k} \pi_{l}(\phi) B_{l} .
$$

As in [10], we define $u$ indirectly. For $\Psi \in C_{c}^{\infty}(N)$, define

$$
\langle\Psi, u\rangle=\int_{V_{2}} \operatorname{Pf}(l)^{k}|\operatorname{Pf}(l)| \operatorname{Tr}\left(B_{l}^{*} \pi_{l}(\phi)^{*} \pi_{l}(\Psi)\right) d_{2} l .
$$

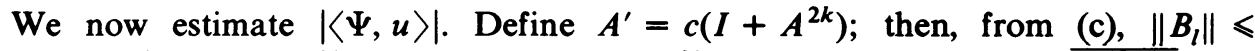
$c|\operatorname{Pf}(l)|^{-k}(1+\operatorname{Pf}(l))^{2 k}, \pi_{l}\left(A^{\prime}\right)=c(1+\operatorname{Pf}(l))^{2 k} I$. Hence, letting $\phi^{\sim}(x)=\overline{\phi\left(x^{-1}\right)}$,

$$
\begin{aligned}
& |\langle\pi, u\rangle| \leqslant \int_{V_{2}}|\operatorname{Pf}(l)|^{k+1}\left|\operatorname{Tr}\left(B_{l}^{*} \pi_{l}(\phi)^{*} \pi_{l}(\Psi)\right)\right| d_{2} l \\
& <\int_{V_{2}}|\operatorname{Pf}(l)|^{k+1}\left\|B_{l}\right\|\left|\operatorname{Tr}\left(\pi_{l}(\phi)^{*} \pi_{l}(\Psi)\right)\right| d_{2} l \\
& <\int_{V_{2}}|\operatorname{Pf}(l)|\left|\operatorname{Tr}\left(\pi_{l}\left(A^{\prime}\right) \pi_{l}\left(\phi^{\sim}\right) \pi_{l}(\Psi)\right)\right| d_{2} l \\
& =\int_{V_{2}}|\operatorname{Pf}(l)|\left|\operatorname{Tr}\left(\pi_{l}\left(A^{\prime} * \phi^{\tilde{y}}\right) \pi_{l}(\Psi)\right)\right| d_{2} l \\
& <\int_{V_{2}}|\operatorname{Pf}(l)|\left\|\pi_{l}\left(A^{\prime} * \phi^{\sim}\right)\right\|_{2}\left\|\pi_{l}(\Psi)\right\|_{2} d_{2} l \\
& <\left(\int_{V_{2}}|\operatorname{Pf}(l)|\left\|\pi_{l}\left(A^{\prime} * \phi^{\tilde{Q}}\right)\right\|_{2}^{2} d_{2} l\right)^{1 / 2}\left(\int_{V_{2}}|\operatorname{Pf}(l)|\left\|\pi_{l}(\Psi)\right\|_{2}^{2} d_{2} l\right)^{1 / 2} \\
& =\left\|A^{\prime} * \phi^{\sim}\right\|_{2}\|\Psi\|_{2} \text {, }
\end{aligned}
$$

where we have used the Cauchy-Schwarz inequality in two different ways (plus the Plancherel formula). Thus $u$ gives a continuous map on $\mathfrak{L}^{2}(N)$, and so $u \in \mathcal{L}^{2}(N)$.

To prove that $u$ is smooth, we need only show that all distribution derivatives of $u$ are in $\mathfrak{L}^{2}$-that is, that $\Psi \rightarrow\langle D * \psi, u\rangle$ is bounded in the \|\|$_{2}$ norm for all 
$D \in \mathcal{U}(\mathscr{N})$. (Then we can apply Sobolev's lemma.) This is easy; we apply the same argument as above, first noting that

$$
\begin{aligned}
\langle D * \psi, u\rangle & =\int_{\mathscr{V}} \operatorname{Pf}(l)^{k}|\operatorname{Pf}(l)| \operatorname{Tr}\left(B_{l}^{*} \pi_{l}\left(\phi^{*}\right) \pi_{l}(D) \pi_{l}(\psi)\right) d_{2} l \\
& =\int_{\mathscr{V}}(\operatorname{Pf}(l))^{k}|\operatorname{Pf}(l)| \operatorname{Tr}\left(B_{l}^{*} \pi_{l}\left(\phi^{\sim} * D\right) \pi_{l}(\psi)\right) d_{2} l .
\end{aligned}
$$

Finally, we check that $u$ is a weak solution of (1), or that $\left\langle u, \Psi * L^{*}\right\rangle=$ $\left\langle A^{k} * \phi, \psi\right\rangle\left(L^{*}=\right.$ formal adjoint of $\left.L\right)$. Since $\pi_{l}\left(\psi * L^{*}\right)=\pi_{l}(\psi) \pi_{l}(L)^{*}$, we have

$$
\begin{aligned}
\left\langle u, \psi * L^{*}\right\rangle & =\int_{\mathscr{V}} \operatorname{Pf}(l)^{k}|\operatorname{Pf}(l)| \operatorname{Tr}\left(B_{l}^{*} \pi_{l}(\phi)^{*} \pi_{l}(\psi) \pi_{l}\left(L^{*}\right)\right) d_{2}(l) \\
& =\int_{\mathscr{V}}|\operatorname{Pf}(l)| \operatorname{Tr}\left(\pi_{l}(A)^{k} \pi_{l}(\phi)^{*} \pi_{l}(\psi) \pi_{l}\left(L^{*}\right) B_{l}^{*}\right) d_{2}(l) \\
& =\int_{\mathscr{V}}|\operatorname{Pf}(l)| \operatorname{Tr}\left(\pi_{l}(\phi)^{*} \pi_{l}\left(A^{*}\right)^{k} \pi_{l}(\psi)\right) d_{2}(l) \\
& =\int_{\mathscr{V}}|\operatorname{Pf}(l)| \operatorname{Tr}\left(\pi_{l}\left(A^{k} * \phi\right)^{*} \pi_{l}(\psi)\right) d_{2}(l) \\
& =\left\langle A^{k} * \phi, \psi\right\rangle
\end{aligned}
$$

by the Plancherel theorem (note that $\pi(A)$ is central and selfadjoint). This proves the theorem.

4. The following remarks may be helpful.

1. Define $L \rightarrow L^{t}$ to be the antiautomorphism of $\mathscr{U}(\mathcal{T})$ which is defined by $L^{t}=-L_{1} \in \mathfrak{N}$. Then $\phi \rightarrow \phi * L$ is the operator usually written as $\phi \rightarrow L^{t} \phi$ (see, e.g., $\$ 2$ of [9]). Let $(T \phi)(x)=\phi\left(x^{-1}\right)=\overline{\phi^{\tilde{}(x)}}$; then

$$
\phi * L^{t}=T(L * T \phi), \quad L^{t} * \phi=T(T \phi * L) .
$$

(These formulas are easy to check for $L \in \Re$ and, by induction, for monomials in थ( $(\Re)$.)

2. Let $\pi_{l}^{t}$ be the representation dual to $\pi_{l}$ (i.e., $\pi_{l}^{t}$ is the contragredient, defined on the linear dual to $\mathcal{H}_{l}$; note that $\mathcal{H}_{l}$ is its own conjugate linear dual). Then $\pi_{l}^{t}=\pi_{-l}$ (as one can see by considering trace characters, for instance), so that $\pi_{l} \in \mathcal{V} \Rightarrow$ $\pi_{l}^{t} \in \mathcal{V} ;$ also,

$$
\pi_{l}^{t}(\phi)=\pi_{l}(T \phi)^{t},
$$

where $S^{t}$ is the (linear) adjoint of $S$. Thus

$$
\begin{aligned}
\pi_{l}\left(L^{t}\right) \pi_{l}(\phi) & =\pi_{l}\left(L^{t} * \phi\right)=\pi_{l}(T(T \phi * L)) \\
& =\left(\pi_{l}^{t}(T \phi * L)\right)^{t}=\left(\pi_{l}^{t}(T \phi) \pi_{l}^{t}(L)\right)^{t} \\
& =\left(\pi_{l}(\phi)^{t} \pi_{l}^{t}(L)\right)^{t}=\left(\pi_{l}^{t}(L)\right)^{t} \pi_{l}(\phi),
\end{aligned}
$$

or $\pi_{l}\left(L^{t}\right)=\left(\pi_{l}^{t}(L)\right)^{t}$, and hypothesis (1) of the theorem is equivalent to the right invertibility of all $\pi_{l}\left(L^{t}\right), l \in \mathcal{V}$.

3. It is now easy to fit the main result of [10] into Theorem 3. That result says that if $N=H_{n}$ and $L$ is homogeneous, then $\phi \rightarrow \phi * L^{t}$ is locally solvable if $\pi_{l}(L)$ 
is right invertible for all $l \in \mathcal{V}$, or, equivalently, if $\pi_{l}\left(L^{t}\right)$ is left invertible. Here, $V_{2} \cong \mathbf{R}$ and $\operatorname{Pf}(l)$ is a power of $l$; the homogeneity assumption means that $\pi_{\lambda l}(L)=\lambda^{s} \pi_{l}(L)$ (for some $s>0$ ) when $\lambda>0$. Thus hypotheses (b) and (c) of Theorem 3 automatically hold.

4. Of course, there is a similar result for $\phi \rightarrow L * \phi$, involving right inverses.

5. One can weaken the hypotheses of Theorem 4 slightly. For instance, suppose that $\pi_{l}(L)^{-1}=B_{l}$ exists for every $l \in \mathcal{V}$ except $l_{0}$, and that the $B_{l}$ also satisfy

$$
\left\|B_{l}\right\| \leqslant c\left|\operatorname{Pf}(l)-\operatorname{Pf}\left(l_{0}\right)\right|^{-k^{\prime}}\left|\operatorname{Pf}(l)^{-k}+\operatorname{Pf}(l)^{k}\right| .
$$

Then $L$ is locally solvable; one simply solves $u * L=A^{k} *\left(A-\operatorname{Pf}\left(l_{0}\right)\right)^{k^{\prime}} * \varphi$ as in the proof of Theorem 3. There are similar theorems in case $\left\|B_{l}\right\|$ is bounded by some other rational function on $V$.

6. The hypothesis of measurability in Theorem 3 is probably unnecessary, in that one can probably always pick left inverses to the $\pi_{l}(A)$ which do vary measurably. I have not tried to prove this, however.

7. Some hypothesis of a growth condition on $\left\|B_{l}\right\|$ is necessary, as the following example shows. Let $\Re$ be the Lie algebra spanned by $W, X, Y$, and $Z$, with $[W, X]=Y,[W, Y]=Z$, and all other brackets 0 (unless given by antisymmetry). Let $N=\exp \Re$. The representations of $N$ in general position are those which are nontrivial on $Z$ (representations killing $Z$ are effectively representations of the Heisenberg group), and those in general position are given on $\pi$ by

$$
\begin{array}{ll}
\sigma_{a, c}(W)=\frac{d}{d t}, & \sigma_{a, c}(X)=2 \pi i\left(c+\frac{a t^{2}}{2}\right), \\
\sigma_{a, c}(Y)=2 \pi i a t, & \sigma_{a, c}(Z)=2 \pi i a I,
\end{array}
$$

where $a, c \in \mathbf{R}$ and $a \neq 0$. Let $\sigma_{c}=\sigma_{a, c}$ with $a=3 / \pi$; we shall examine $\sigma_{c}(W+i X) . W+i X$ is not locally solvable, since $[W, X]$ is independent of $W$ and $X$; see Theorem 6.1.1 of [11]. However,

$$
\sigma_{c}(W+i X) \phi=\frac{d \phi}{d t}-\left(2 \pi c+3 t^{2}\right) \phi .
$$

For simplicity, let $2 \pi c=\alpha$, and define

$$
\left(A_{\alpha} f\right)(t)=-\int_{t}^{\infty} f(s) \exp \left(t^{3}-s^{3}+\alpha t-\alpha s\right) d s .
$$

Then $\phi=A_{\alpha} f$ solves $d / d t-\left(3 t^{2}+\alpha\right) \phi=f$. Moreover, $A_{\alpha}$ is a Hilbert-Schmidt operator on $\mathcal{L}^{2}(R)$, because

$$
\begin{aligned}
\int_{-\infty}^{\infty} \int_{t}^{\infty} \exp & -2\left(t^{3}-s^{3}+\alpha t-\alpha s\right) d s d t \\
= & \int_{-\infty}^{\infty} \int_{0}^{\infty} \exp \left(-2 u-6 u t^{2}-6 u^{2} t-2 u\right) \\
= & \int_{-\infty}^{\infty} \int_{0}^{\infty} \exp (-2 \alpha u) \exp \left(-6 u t^{2}-6 u^{2} t-2 u^{3}\right) d u d t \\
= & \int_{0}^{\infty} \int_{-\infty}^{\infty} \exp \left(-2 \alpha u-\frac{u^{3}}{2}\right) \exp \left[(-6 u)\left(t+\frac{u}{2}\right)^{2}\right] d t d u \\
= & \int_{0}^{\infty}\left(\frac{\pi}{6 u}\right)^{1 / 2} \exp \left(-2 \alpha u-\frac{1}{2} u^{3}\right) d u<\infty
\end{aligned}
$$


However, from the equation,

$$
\left(A_{\alpha} f\right)(t)=-\int_{0}^{\infty} f(t+u) \exp \left(-3 u t^{2}-3 u^{2} t-u^{3}\right) \exp (-\alpha u) d u
$$

Let $f$ be the characteristic function of $[0,2]$. Then the integrand is nonnegative, and is positive if $0 \leqslant t \leqslant 1$ and $1 \leqslant u \leqslant 2$. It is clear that on this interval, $\left(A_{a} f\right)(t) \geqslant$ $C e^{-\alpha}$ for some $C>0$; thus $\left\|A_{\alpha}\right\| \geqslant C^{\prime} e^{-\alpha}$ for some $C^{\prime}>0$. Hence $\left\|A_{\alpha}\right\|$ is not polynomially bounded as $\alpha \rightarrow-\infty$.

8. The proof of Theorem 3 actually shows that the operator $L$ is semiglobally solvable.

9. I am indebted to Professor L. P. Rothschild for several valuable conversations.

\section{BIBLIOGRAPHY}

1. L. Corwin and F. P. Greenleaf, Character formulas and spectra of compact nilmanifolds, J. Funct. Anal. 21 (1976), 123-154.

2. J. Dixmier, Algèbres enveloppantes, Gauthier-Villars, Paris, 1974.

3. M. Duflo, Caractères des groupes et des algèbres de Lie resolubles, Ann. Sci. École Norm. Sup. (4) 3 (1970), 23-74.

4. A. A. Kirillov, Unitary representations of nilpotent Lie groups, Uspehi Mat. Nauk 17 (1962), 57-110; English transl. in Russian Math. Surveys.

5. C. C. Moore, Representations of solvable and nilpotent groups and harmonic analysis on nil and submanifolds, Harmonic Analysis on Homogeneous Spaces, Proc. Sympos. Pure Math., vol. 26, Amer. Math. Soc., Providence, R. I., 1974, pp. 3-44.

6. L. Pukanszky, Lecons sur les représentations des groupes, Dunod, Paris, 1967.

7. , On the characters and the Plancherel formula of nilpotent groups, J. Funct. Anal. 1 (1967), 255-280.

8. M. Rais, Solutions elementaires des opérateurs differentials bi-invariants sur un groupe de Lie nilpotent, C. R. Acad. Sci. Paris Ser. A-B 273 (1971), A495-A498.

9. C. Rockland, Hypoellipticity on the Heisenberg group-Representation-theoretic criteria, Trans. Amer. Math. Soc. 240 (1978), 1-52.

10. L. Rothschild, Local solvability of left invariant differential operators on the Heisenberg group, Proc. Amer. Math. Soc. 74 (1979), 383-388.

11. Lars Hörmander, Linear partial differential operators, Die Grundlehren der Math. Wissenschaften, Bd. 116, Springer-Verlag, Berlin and New York, 1963.

Department of Mathematics, Rutgers University, New Brunswick, New Jersey 08903 